April 27, 2001

\title{
Differential Geometry from Differential Equations
}

\author{
Simonetta Frittelli \\ Dept of Physics, Duquesne Univ., Pgh. PA15282 \\ Dept of Physics, Univ. of Pittsburgh, Pgh., PA15260 \\ Carlos Kozameh \\ FaMAF, Universidad Nacional de Cordoba, 500 Cordoba, Argentina \\ Ezra T. Newman \\ Dept of Physics and Astronomy, \\ Univ. of Pittsburgh, Pittsburgh, PA 15260
}

\begin{abstract}
We first show how, from the general 3rd order ODE of the form $z^{\prime \prime \prime}=F\left(z, z^{\prime}, z^{\prime \prime}, s\right)$, one can construct a natural Lorentzian conformal metric on the four-dimensional space $\left(z, z^{\prime}, z^{\prime \prime}, s\right)$. When the function $F\left(z, z^{\prime}, z^{\prime \prime}, s\right)$ satisfies a special differential condition the conformal metric possesses a conformal Killing field, $\xi=\partial / \partial s$, which in turn, allows the conformal metric to be mapped into a three dimensional Lorentzian metric on the space $\left(z, z^{\prime}, z^{\prime \prime}\right)$ or equivalently, on the space of solutions of the original differential equation. This construction is then generalized to the pair of differential equations, $z_{s s}=S\left(z, z_{s}, z_{t}, z_{s t}, s, t\right)$ and $z_{t t}=$ $T\left(z, z_{s}, z_{t}, z_{s t}, s, t\right)$, with $z_{s}$ and $z_{t}$ the derivatives of $z$ with respect to $s$ and $t$. In this case, from $S$ and $T$, one can again, in a natural manner, construct a Lorentzian conformal metric on the six dimensional space $\left(z, z_{s}, z_{t}, z_{s t}, s, t\right)$. When the $S$ and $T$ satisfy differential conditions analogous to those of the 3rd order ode, the 6 space then possesses a pair of conformal Killing fields, $\xi=\partial / \partial s$ and $\eta=\partial / \partial t$
\end{abstract}


which allows, via the mapping to the four-space of $\left(z, z_{s}, z_{t}, z_{s t}\right)$ and a choice of conformal factor, the construction of a four-dimensional Lorentzian metric. In fact all four- dimensional Lorentzian metrics can be constructed in this manner. This construction, with further conditions on $S$ and $T$, thus includes all (local) solutions of the Einstein equations. 


\section{Introduction}

For the last 10 or so years we have been developing and studying a reformulation of General Relativity where the primary objects of study are families of 3-dimensional surfaces in a 4-space. They are used to define a conformal Lorentzian metric via the requirement that the surfaces be null or characteristic surfaces of that conformal metric; with, in addition, the choice of a conformal factor to make the conformal metric into an Einstein metric. From this point of view the metric tensor is a derived concept and the Einstein equations appear as equations for the surfaces and the conformal factor. In this study it had on occasion been useful to simplify some of the equations by assuming we were studying conformal geometry in a three-dimensional Lorentzian manifold. Much to our surprise, we recently discovered that this 3-dimensional problem - with a totally different motivation - had been studied in some classical papers by Cartan and Chern. One major purpose of this work is to examine the relationship of our version of 3-dimensional Lorentzian conformal geometry with that of Cartan and Chern. Of perhaps greater importance is our generalization of these ideas to the case of 4-dimensional conformal Lorentzian geometries. This, very much more complicated, problem is discussed after an exposition of the 3dimensional problem. Though one might consider these investigations to be mainly in the realm of the study of certain classes of differential equations, our main motivation has been towards the investigation of the Einstein equations of general relativity. Already the theory of self- (or anti-) dual vacuum Einstein metrics has arisen as a natural special case. The emphasis now is on the full vacuum case.

In the late 1930's Cartan and Chern, properties of differential equations, showed that there was a natural geometric structure that can be associated with ordinary differential equations [ODE's] of the form $z^{\prime \prime}=E\left(z, z^{\prime}, s\right)$ or $z^{\prime \prime \prime}=F\left(z, z^{\prime}, z^{\prime \prime}, s\right)$ where the prime denotes differentiation with respect to the independent variable $s$. This geometric structure which is quite rich, involving a wide variety of connections (projective, conformal and metric with and without torsion), is given on the solution spaces of the equations.

More specifically, if the solutions are denoted by $z=z\left(x^{a}, s\right)$ where $x^{a}$ are the arbitrary constants of integration, (two of them for the first equation and three for the second) the geometric structures often (or usually) live on the 
space of the constants of integration, but are sometimes augmented with an extra dimension by adding the independent variable $s$ as a fiber coordinate.

In Section II, we first review, from a new perspective, these results of Cartan and Chern applied to the third order equation, and then, in Section III, we generalize them to a new system of equations. For the new system we will consider the dependent variable, $z$, to be a function of now two independent variables, $s$ and $t$, e.g., $z=z(s, t)$ that satisfies the system of equations $z_{s s}=S\left(z, z_{s}, z_{t}, z_{s t}, s, t\right)$ and $z_{t t}=T\left(z, z_{s}, z_{t}, z_{s t}, s, t\right)$, with $z_{s}$ and $z_{t}$ the derivatives of $z$ with respect to $s$ and $t$. The integrability conditions, $S_{t t}=T_{s s}$, are assumed to be satisfied. The solution space to these equations, which is four-dimensional and is again augmented by the fiber coordinates $s$ and $t$, possesses a natural six-dimensional conformal metric. By a judicious choice of the two functions, $S$ and $T$, (i.e. by being solutions of a complicated differential equation) the six-dimensional conformal metric possesses two conformal Killing fields and (via them and a special choice of conformal factor) maps to a family of conformal Lorentzian metrics on the four-dimensional solution space. All four-dimensional Lorentzian metrics can be obtained in this manner. It follows that by a further restriction in the choice of $S$ and $T$ and choice of conformal factor, all Einstein spaces can be so obtained. In Section IV we discuss the relationship of this work to the earlier work on the null surface reformulation of GR.

For the sake of completeness, in Appendix A, we will outline the Cartan geometry associated with the second order equation, $z^{\prime \prime}=E\left(z, z^{\prime}, s\right)$.

\section{The Differential Geometry of $z^{\prime \prime \prime}=F\left(z, z^{\prime}, z^{\prime \prime}, s\right)$}

We will study the geometry associated with the differential equation $z^{\prime \prime \prime}=F\left(z, z^{\prime}, z^{\prime \prime}, s\right)$ assuming that $F\left(z, z^{\prime}, z^{\prime \prime}, s\right)$ is a smooth function in all its variables. We will only be interested in the local behavior of the solutions. There will be several different (but related) spaces that will be of interest to us. First of all, we mention the two-dimensional space of $(z, s)$; Cartan and Chern studied the problem of the equivalence classes of differential equations under the diffeomorphisms in this two-space. This problem, though of considerable interest, will not concern us. The next space is the three-dimensional solution space of the differential equation. The solutions of the third order ODE are given in terms of three constants of integration, $x^{a}$, so that $z=z\left(x^{a}, s\right)$ is the general solution and the space of the $x^{a}$ is 
the solution space. For any fixed value of the independent variable $s$, the relations

$$
z=z\left(x^{a}, s\right), \quad z^{\prime}=z^{\prime}\left(x^{a}, s\right), \quad z^{\prime \prime}=z^{\prime \prime}\left(x^{a}, s\right)
$$

can be considered as a coordinate transformation (that depends on the parameter $s$ ), between the three $x^{a}$ and the "coordinates" $\left(z, z^{\prime}, z^{\prime \prime}\right)$, i.e., it defines a one-parameter family of coordinate transformations. This leads naturally to the idea of a four-dimensional space coordinatized either by $\left(x^{a}, s\right)$ or by $\left(z, z^{\prime}, z^{\prime \prime}, s\right)$. The first choice suggests that this four-space should be thought of as a base three-space augmented by the one-dimensional fibers coordinatized by $s$. Different constructions or applications lead naturally to one or the other of the coordinatizations.

Remark 1 For most applications that are of interest to us, the independent variable $s$ is taken to be the angle $\phi$ on the circle and the fiber would thus be thought of as $S^{1}$. This circle, in our applications to 3-dimensional Lorentzian spaces, is simply the "circle" of null directions at each space-time point. At this point in the exposition, this, however, is not easily seen. Here, for the moment, we are only interested in the local behavior.

On the four dimensional space of $\left(z, z^{\prime}, z^{\prime \prime}, s\right)$, we consider the four oneforms $\beta^{a}$,

$$
\begin{aligned}
& \beta^{1}=d z-z^{\prime} d s \\
& \beta^{2}=d z^{\prime}-z^{\prime \prime} d s, \\
& \beta^{3}=d z^{\prime \prime}-F\left(z, z^{\prime}, z^{\prime \prime}, s\right) d s, \\
& \beta^{4}=d s .
\end{aligned}
$$

From (1), we can write

$$
\begin{aligned}
d z & =\partial_{a} z d x^{a}+z^{\prime} d s \\
d z^{\prime} & =\partial_{a} z^{\prime} d x^{a}+z^{\prime \prime} d s \\
d z^{\prime \prime} & =\partial_{a} z^{\prime \prime} d x^{a}+F\left(z, z^{\prime}, z^{\prime \prime}, s\right) d s
\end{aligned}
$$

so that we have the alternative version of the forms

$$
\begin{aligned}
& \beta^{1}=z_{a} d x^{a}, \\
& \beta^{2}=z_{a}^{\prime} d x^{a}, \\
& \beta^{3}=z_{a}^{\prime \prime} d x^{a}, \\
& \beta^{4}=d s .
\end{aligned}
$$


The following four linear combinations of the $\beta$ 's will play a central role, though for the moment only the first three will be used,

$$
\begin{aligned}
\omega^{1} & =\beta^{1}, \\
\omega^{2} & =\beta^{2}, \\
\omega^{3} & =\beta^{3}+a \beta^{1}+b \beta^{2}, \\
\omega^{4} & =C \beta^{4} .
\end{aligned}
$$

The $(a, b, C)$ are three functions of $\left(z, z^{\prime}, z^{\prime \prime}, s\right)$ that are to be determined.

From the $\omega^{i}=\left(\omega^{1}, \omega^{2}, \omega^{3}\right)$ we construct the following one-parameter family of Lorentzian 3-metrics, parametrized by the values of $s$;

$$
g\left(z, z^{\prime}, z^{\prime \prime}, s\right)=\omega^{1} \otimes \omega^{3}+\omega^{3} \otimes \omega^{1}-\omega^{2} \otimes \omega^{2} .
$$

At this point we are simply defining a one-parameter family of metrics constructed from the $\left(\omega^{1}, \omega^{2}, \omega^{3}\right)$. Later we will see that this definition is justified by the results.

Note: A more general version of (4) could have been used. The $\omega^{2}$ could have included another term so that it had the form $\omega^{2}=\beta^{2}+A \beta^{1}$ with the further modification $\omega^{3}=\beta^{3}+(A+a) \beta^{1}+\left(\frac{1}{2} A^{2}+b\right) \beta^{2}$. These exta terms however play no role; they form a "null" rotation, leaving the metric, (5) invariant with arbitrary $A$.)

Remark 2 In order to try to give some perspective and motivation we remark that from another point of view, described in detail later, the metric (5) arose from physical considerations where the function

$u=z\left(x^{a}, s\right)=$ const., represented a one-parameter family of null foliations of a Lorentzian three-dimensional space-time. The three $\omega$ 's are chosen to form a null triad with $\omega^{1}$ being the gradient of $z\left(x^{a}, s\right)$ and with $\omega^{3}$ as the second null covector of the triad. The (so-far) arbitrary functions a and $b$ will be uniquely determined by a requirement of "minimal" dependence of the metric, Eq.(5), on the parameter s. The precise meaning of " "minimal" dependence' will be given shortly.

Remark 3 We emphasize that, though it appears that our choice of the form of the metric Eq. (5) is arbitrary, in fact, it appears to be the only choice that allows the following construction. Later we will give, from the other point of view, an alternate justification of its "naturalness". 
First we have:

Definition 1 For any function $H\left(z, z^{\prime}, z^{\prime \prime}, s\right), \dot{H}$ is the total s-derivative;

$$
\frac{d H}{d s} \equiv \dot{H} \equiv H_{z} z^{\prime}+H_{z^{\prime}} z^{\prime \prime}+H_{z^{\prime \prime}} F+H,_{s}
$$

Our plan is to first take the $s$-derivative of $g\left(z, z^{\prime}, z^{\prime \prime}, s\right)$, i.e.,

$$
\dot{g} \equiv \frac{d g\left(z, z^{\prime}, z^{\prime \prime}, s\right)}{d s},
$$

and then by judicious choice of $a$ and $b$, to make $\dot{g}$ as close as possible to being proportional to $g$ itself.

Remark 4 This derivative is actually the Lie derivative of the metric along the vector field,

$$
\frac{d}{d s} \equiv \frac{\partial}{\partial s}+z^{\prime} \frac{\partial}{\partial z}+z^{\prime \prime} \frac{\partial}{\partial z^{\prime}}+F \frac{\partial}{\partial z^{\prime \prime}},
$$

in the $\left(z, z^{\prime}, z^{\prime \prime}, s\right)$ space. It is, perhaps, simpler to think of it as the total $s$-derivative of $g$ in the $\left(z, z^{\prime}, z^{\prime \prime}, s\right)$ coordinate system.

It turns out that to exactly make $\dot{g}=\lambda g$ requires a restriction on the $F$ of the starting differential equation, $z^{\prime \prime \prime}=F\left(z, z^{\prime}, z^{\prime \prime}, s\right)$. Nevertheless it is the "as close as possible" condition that will constitute our "minimal" dependence' condition. Note that when $\dot{g}=\lambda g$ there is a conformal structure naturally defined on the solution space.

Explicitly the $s$-derivative of $g\left(z, z^{\prime}, z^{\prime \prime}, s\right)$, is

$$
\dot{g}=\dot{\omega}^{1} \otimes \omega^{3}+\omega^{1} \otimes \dot{\omega}^{3}+\dot{\omega}^{3} \otimes \omega^{1}+\omega^{3} \otimes \dot{\omega}_{1}-\dot{\omega}^{2} \otimes \omega^{2}-\omega^{2} \otimes \dot{\omega}^{2}
$$

From the definition of the forms we have for the first two $\omega$ that

$$
\begin{aligned}
& \dot{\omega}^{1}=\omega^{2}, \\
& \dot{\omega}^{2}=\omega^{3}-a \omega^{1}-b \omega^{2},
\end{aligned}
$$

Using 


$$
\begin{aligned}
\dot{\omega}^{3} & =\dot{\beta}^{3}+\dot{a} \omega^{1}+a \dot{\omega}^{1}+\dot{b} \omega^{2}+b \dot{\omega}^{2} \\
\dot{\beta}^{3} & =z_{a}^{\prime \prime \prime} d x^{a}=F_{a} d x^{a}=F_{z} \omega^{1}+F_{z^{\prime}} \omega^{2}+F_{z^{\prime \prime}}\left(\omega^{3}-a \omega^{1}-b \omega^{2}\right)
\end{aligned}
$$

we have

$$
\dot{\omega}_{3}=\left(F_{z}-a F_{z^{\prime \prime}}+\dot{a}-a b\right) \omega^{1}+\left(F_{z^{\prime}}-b F_{z^{\prime \prime}}+a+\dot{b}-b^{2}\right) \omega^{2}+\left(F_{z^{\prime \prime}}+b\right) \omega^{3}
$$

or

$$
\dot{\omega}_{3}=U \omega^{1}+V \omega^{2}+W \omega^{3}
$$

with

$$
\begin{aligned}
U & =F_{z}-a F_{z^{\prime \prime}}+\dot{a}-a b, \\
V & =F_{z^{\prime}}-b F_{z^{\prime \prime}}+a+\dot{b}-b^{2}, \\
W & =F_{z^{\prime \prime}}+b .
\end{aligned}
$$

Substituting Eqs.(7) and (8) into Eq.(6) we obtain, after collecting terms,

$$
\dot{g}=2 U \omega^{1} \otimes \omega^{1}+2(V+a) \omega^{(1} \otimes \omega^{2)}+2 W \omega^{(1} \otimes \omega^{3)}+2 b \omega^{2} \otimes \omega^{2} .
$$

We can now precisely state our condition of "minimal $s$ dependence" of the metric;

1. We require that, in Eq.(10), the coefficient of $\omega^{(1} \otimes \omega^{2)}$ vanishes, i.e.,

$$
a=-V,
$$

2. We require the two terms, $2 W \omega^{(1} \otimes \omega^{3)}+2 b \omega^{2} \otimes \omega^{2}$, combine so that they are proportional to the metric, Eq.(5), i.e.,

$$
2 b=-W .
$$

This leads to the unique algebraic determination of $a$ and $b$ in terms of $F$ and its derivatives: 


$$
\begin{aligned}
b & =-\frac{1}{3} F_{z^{\prime \prime}}, \\
2 a & =-F_{z^{\prime}}-\frac{2}{9}\left(F_{z^{\prime \prime}}\right)^{2}+\frac{1}{3} \frac{d}{d s}\left(F_{z^{\prime \prime}}\right) .
\end{aligned}
$$

Using, from Eqs.(9) and (13),

$$
U[F] \equiv F_{z}-a[F] F_{z^{\prime \prime}}+\dot{a}[F]-a[F] b[F],
$$

this leads to the final form of $\dot{g}$ :

$$
\dot{g}[F]=2 U[F] \omega^{1} \otimes \omega^{1}+\lambda g,
$$

with

$$
\lambda\left(x^{a}, s\right)=\frac{2}{3} F_{z^{\prime \prime}}
$$

Our "minimal $s$ dependence" leads to a unique determination of $a$ and $b$ and unique differential expressions for both $U$ and $\lambda$ in terms of $F$.

Proposition 2 Our one-parameter family of metrics are all conformally related if $F$ is restricted by the condition $U[F]=0$, so that

$$
\dot{g}[F]=\frac{2}{3} F_{z^{\prime \prime}} g .
$$

In this case there exists a conformal factor, $\Omega$, with $\dot{\Omega}=\frac{1}{3} F_{z^{\prime \prime}} \Omega$, so that, for all values of $s$, the metric $\widehat{g}=\Omega^{-2} g$, satisfies $\frac{d}{d s} \widehat{g}=0$.

In the general case, $U[F] \neq 0$, we can extend the metric $g$ to a four dimensional metric by

$$
g^{(4)}=g-\omega^{4} \otimes \omega^{4}=g-C^{2} d s \otimes d s
$$

so that

$$
\begin{aligned}
\dot{g}^{(4)} & =\dot{g}-2 C \dot{C} d s \otimes d s, \\
& =2 U \omega^{1} \otimes \omega^{1}+\frac{2}{3} F_{z^{\prime \prime}} g-2 C \dot{C} d s \otimes d s,
\end{aligned}
$$

If the unknown $C$ is chosen such that $\dot{C}=\frac{1}{3} F_{z^{\prime \prime}} C$ then

$$
\dot{g}^{(4)}=2 U \omega^{1} \otimes \omega^{1}+\frac{2}{3} F_{z^{\prime \prime}} g^{(4)} .
$$


Proposition 3 For the special case of $U[F]=0$, we see that $\xi=d / d s$ is a conformal Killing field of the four-space so that each of the "three-slices", $s=$ constant, yield three-metrics that are conformally related. If $g^{(4)}$ is conformally rescaled by $\widehat{g}^{(4)}=\Omega^{-2} g^{(4)}$, with $\dot{\Omega}=\frac{1}{3} F_{z^{\prime \prime}} \Omega$, the conformal Killing vector field becomes a Killing field and the three-slices are all isometric.

An alternate point of view towards the geometry of $z^{\prime \prime \prime}=F\left(z, z^{\prime}, z^{\prime \prime}, s\right)$ is via the first of Cartan's structure equations, for the three one-forms

$$
\omega^{i}=\left(\omega^{1}, \omega^{2}, \omega^{3}\right)
$$

we have;

$$
d \omega^{i}=\omega_{j}^{i} \wedge \omega^{j}+T^{i}
$$

The indices are raised and lowered with the Lorentzian metric, (from Eq.(5)), $\eta_{i j}$, with $\eta_{13}=-\eta_{22}$, all other independent components vanishing. The basis one-forms are taken as $d x^{a}$ and $d s$; so that though the $\omega^{i}$ contain only $d x^{a}$, the other forms, i.e., $d \omega^{i}, \omega_{j}^{i}$ and $T^{i}$ will, in general, contain $d s$. The connection one-forms, $\omega^{i}{ }_{j}$, do not form a metric connection but rather a conformal connection. Written as $\omega_{i j}=\eta_{i k} \omega_{j}^{k}$ they are given by

$$
\begin{aligned}
\omega_{i j} & =w_{i j}+\widehat{\omega} \eta_{i j}, \\
w_{i j} & =-w_{j i}
\end{aligned}
$$

i.e., are taken as a metric connection plus a trace-term, $\left(\widehat{\omega}=\frac{1}{3} \omega_{i}^{i}\right)$.

Remark 5 Note that the use of the trace term in the connection, $\omega_{i j}$, is a variant of a (Weyl) connection via the equation $\nabla_{c} g_{a b}\left(x^{a}\right)=2 \widehat{\omega}_{c} g_{a b}$. It is not exactly the same as a Weyl connection, but is a variant of it, because here we have the extra degree of freedom, namely the variable s.

Writing out Eq.(19) we have

$$
\begin{aligned}
d \omega^{1} & =\left(w_{[31]}+\widehat{\omega}\right) \wedge \omega^{1}+w_{[32]} \wedge \omega^{2}+T^{1}, \\
d \omega^{2} & =-w_{[21]} \wedge \omega^{1}+\widehat{\omega} \wedge \omega^{2}-w_{[23]} \wedge \omega^{3}+T^{2}, \\
d \omega^{3} & =w_{[12]} \wedge \omega^{2}+\left(w_{[13]}+\widehat{\omega}\right) \wedge \omega^{3}+T^{3}
\end{aligned}
$$


which, to determine the structure and torsion forms, can be compared with the direct calculation of $d \omega^{i}$, namely

$$
\begin{aligned}
d \omega^{1}= & d s \wedge \omega^{2} \\
d \omega^{2}= & d s \wedge\left(\omega^{3}-a \omega^{1}-b \omega^{2}\right) \\
d \omega^{3}= & d s \wedge\left(\omega^{1} U[F]-a \omega^{1}-2 b \omega^{2}\right) \\
& +\left(a_{z^{\prime}}-b a_{z^{\prime \prime}}-b_{z}+a b_{z^{\prime \prime}}\right) \omega^{2} \wedge \omega^{1}+a_{z^{\prime \prime}} \omega^{3} \wedge \omega^{1}+b_{z^{\prime \prime}} \omega^{3} \wedge \omega^{2} .
\end{aligned}
$$

When the comparison is made we see that there are far more variables than equations and thus there are ambiguities in the algebraic solution for $w_{i j}, \widehat{\omega}$ and $T^{i}$. If however we require that the skew-part of the connection, when pulled back to the constant $s$ surfaces, be precisely the metric connection of Eq.(5), then we have a unique solution for the connection and torsion:

$$
\begin{aligned}
w_{[32]} & =d s-\frac{1}{2} b_{z^{\prime \prime}} \omega^{1}, \\
w_{[31]} & =-a_{z^{\prime \prime}} \omega^{1}-\frac{1}{2} b_{z^{\prime \prime}} \omega^{2}+b d s, \\
w_{[12]} & =-a d s+\left(a_{z^{\prime}}-b a_{z^{\prime \prime}}-b_{z}+a b_{z^{\prime \prime}}\right) \omega^{1}-\frac{1}{2} b_{z^{\prime \prime}} \omega^{3}, \\
\widehat{\omega} & =-b d s, \quad T^{2}=0, \quad T^{3}=U[F] d s \wedge \omega^{1} .
\end{aligned}
$$

Once again we see the geometric role of $U[F]$; when it vanishes the "conformal" connection has zero torsion.

We thus have seen that the general third order differential equation

$$
z^{\prime \prime \prime}=F\left(z, z^{\prime}, z^{\prime \prime}, s\right)
$$

induces a variety of geometric structures; a "conformal" connection on the solutions space, $x^{a}$, a four-dimensional Lorentzian metric on the space $\left(z, z^{\prime}, z^{\prime \prime}, s\right)$ so that when the space is foliated by the constant $s$ three-surfaces they possess a one parameter family of three-metrics, all closely related, satisfying $\dot{g}=2 U[F] \omega^{1} \otimes \omega^{1}+\frac{2}{3} F_{z^{\prime \prime}} g$. When the special condition $U[F]=0$ is satisfied all the three-metrics are conformally equivalent. Cartan studied the connection associated with the full conformal equivalence class. We, instead, worked out the metric connections, Eq.(24), associated with the one-parameter family of metrics, Eq.(5). 
Remark 6 The study, 国四5 of this third order ODE had its origin in the classical question of the equivalence of $O D E$ 's under transformations in the plane; $(z, s) \Leftrightarrow\left(z^{*}, s^{*}\right)$. Cartan studied the equivalence classes (with their invariants) of $3 r d$ order ODEs under point transformations, $z^{*}=\mathfrak{Z}(z, s)$, $s^{*}=\mathfrak{S}(z, s)$, while Chern studied the same problems but under a larger group of transformation, the group of contact transformations. The functional $U[F]$, often referred as the Wunschmann Invariant, is a relative invariant under contact transformations of the 3rd order ODEs. We return briefly to this issue in the Discussion section.

To conclude this section we will summarize, 13,14 what appears to be a completely different problem that in fact turns out to be virtually identical, or more correctly, turns out to be the inverse to the problem just addressed, namely the geometry of 3rd order ODEs. Roughly speaking, we begin with 3-dimensional conformal Lorentzian metric and find a complete integral of the associated Eikonal equation; i.e., we find a one parameter, 's', family of characteristic surfaces of sufficient generality. By taking three derivatives with respect to the parameter, the three space-time coordinates can be eliminated from the eikonal, resulting in a 3rd order ODE with ' $\mathrm{s}$ ' being the independent variable. Automatically the Wunschmann Invariant vanishes. Actually, (along with the problem of the four-dimensional solution space of the next section) this inverse point of view was how we first addressed the issues of this work.

More precisely, we begin with a three manifold, $\mathfrak{M}$, locally coordinatized by, $x^{a}$, and require that there be a Lorentzian (conformal) metric determined in the following manner: there is to exist a one-parameter family of foliations of $\mathfrak{M}$, of sufficient generality, (referred to as a complete integral) such that every member of the foliation is to be a null-surface of the unknown metric. If the level surfaces of the one-parameter family of foliations (parametrized by $s$ ) is given by

$$
u=z\left(x^{a}, s\right),
$$

then the condition that they be null, for all values of $s$, with respect to the unknown metric, $g^{a b}\left(x^{a}\right)$, is

$$
g^{a b} \partial_{a} z \partial_{b} z \equiv g^{a b} z_{a} z_{b}=0 .
$$

By now taking a series (four) of $s$ derivatives of Eq.(25), we have 


$$
\begin{aligned}
g^{a b} z_{a}^{\prime} z_{b} & =0, \\
g^{a b} z_{a}^{\prime \prime} z_{b}+g^{a b} z_{a}^{\prime} z_{b}^{\prime} & =0, \\
g^{a b} z_{a}^{\prime \prime \prime} z_{b}+3 g^{a b} z_{a}^{\prime \prime} z_{b}^{\prime} & =0, \\
g^{a b} z_{a}^{\prime \prime \prime \prime} z_{b}+4 g^{a b} z_{a}^{\prime \prime \prime} z_{b}^{\prime}+3 g^{a b} z_{a}^{\prime \prime} z_{b}^{\prime \prime} & =0 .
\end{aligned}
$$

Then by considering the set

$$
u=z\left(x^{a}, s\right), \quad w=z^{\prime}\left(x^{a}, s\right), \quad R=z^{\prime \prime}\left(x^{a}, s\right), \quad F=z^{\prime \prime \prime}\left(x^{a}, s\right)
$$

the three $x^{a}$ can be eliminated from the last expression via the first three expressions, yielding

$$
z^{\prime \prime \prime}=F\left(z, z^{\prime}, z^{\prime \prime}, s\right) .
$$

From the form of the metric, (5), we have that $\omega^{1}=z_{a} d x^{a}$ is a null covector. This observation thus establishes the connection with the first approach.

[Note that by "a one parameter family of sufficient generality", we mean that the three one-forms $\left(z_{a} d x^{a}, z_{a}^{\prime} d x^{a}, z_{a}^{\prime \prime} d x^{a}\right)$ are linearly independent for all s. This implies that one can invert (30), i.e., obtain $x^{a}=X^{a}\left(z, z^{\prime}, z^{\prime \prime}, s\right)$.]

We see that, via Eq.(31), $z_{a}^{\prime \prime \prime}$ and $z_{a}^{\prime \prime \prime \prime}$ can be expressed in terms of the gradient basis $\left(z_{a}, z_{a}^{\prime}, z_{a}^{\prime \prime}\right)$. The five expressions Eqs. (25) and (26) yield the five independent components of a conformal metric that depends on $s$. This conformal metric (though described in the gradient basis) is identical to the conformal metric of Eq.(5) which is described in a null basis.

The fifth derivative of Eq. 251), namely

$$
g^{a b} z_{a}^{(5)} z_{b}+5 g^{a b} z_{a}^{(4)} z_{b}^{\prime}+10 g^{a b} z_{a}^{\prime \prime \prime} z_{b}^{\prime \prime}=0,
$$

when expressed in terms of $F$ and its derivatives, is identical to Eq.(14), i.e., $U[F]=0$. This completes the display of the equivalence of the two approaches. It also gives the justification for the apparently arbitrary choices of the one-forms (4) and metric (5). Linear combinations of the three gradient one-forms, $\left(z_{a} d x^{a}, z_{a}^{\prime} d x^{a}, z_{a}^{\prime \prime} d x^{a}\right)$, form the null triad $\left(\omega^{1}, \omega^{2}, \omega^{3}\right)$.

In addition to the condition $U[F]=0$, Tod, following Cartan, 目 in a continuing study of the Eq.(31) imposes further restrictions on $F$ so that the resulting metrics contain all three-dimensional Einstein -Weyl spaces. 


\section{Pairs of Partial Differential Equations}

The discussion of the previous section is really a variant of the work of Cartan and Chern with our point of view. In this section we will discuss a new situation. We want to find differential equations whose solution space is four-dimensional and in addition possess a Lorentzian structure. This four-dimensional solution space is to be the four-dimensional manifold $\mathfrak{M}$ of physical space-time. Our goal, eventually, is to impose the Einstein vacuum equations on this space. This issue however will not be addressed here. After the consideration of the equation $z^{\prime \prime \prime}=F\left(z, z^{\prime}, z^{\prime \prime}, s\right)$ one might have thought that the generalization from three to four dimensions should be to an equation of the form, $z^{\prime \prime \prime \prime}=G\left(z, z^{\prime}, z^{\prime \prime}, z^{\prime \prime \prime}, s\right)$ whose solution space is four dimensional. This case was studied by Bryant who found a further rich variety of geometric structures, e.g., a quartic metric, $g_{a b c d}$ but it does not include a four-dimensional Lorentzian structure.

We have taken a different direction for the creation of a four-dimensional solution space; we consider and study the geometry of the pair of equations

$$
Z_{s s}=P\left(Z, Z_{s}, Z_{t}, Z_{s t}, s, t\right), Z_{t t}=Q\left(Z, Z_{s}, Z_{t}, Z_{s t}, s, t\right),
$$

where $P$ and $Q$ satisfy the integrability conditions for all $Z$,

$$
D_{t}^{2} P=D_{s}^{2} Q
$$

and the weak inequality, needed for the four-dimensionality of the solution space,

$$
1>\left(\frac{\partial P}{\partial Z_{t s}}\right)\left(\frac{\partial Q}{\partial Z_{t s}}\right) .
$$

We have used the notation for the total derivatives $D_{t}$ or $D_{s}$ to mean, respectively, the $t$ and $s$ derivatives acting on all the variables but holding, respectively, the $s$ or the $t$ constant. For example, if $H=H\left(Z, Z_{s}, Z_{t}, Z_{s t}, s, t\right)$ then

$$
D_{t} H \equiv \frac{\partial H}{\partial Z} Z_{t}+\frac{\partial H}{\partial Z_{s}} Z_{t s}+\frac{\partial H}{\partial Z_{t}} Z_{t t}+\frac{\partial H}{\partial Z_{t s}} Z_{t t s}+\frac{\partial H}{\partial t}
$$

$D_{t}$ and $D_{s}$ should also be thought of as vector fields on the six-space, $\left(Z, Z_{s}, Z_{t}, Z_{s t}, s, t\right)$. 
For notational reasons and for comparison with earlier work but without changing anything essential, we will consider $P$ and $Q$ to be complex conjugates of each other, $s$ and $t$ to also be complex conjugates of each other and will adopt the notation that $P=S$ and $Q=S^{*}$ and $t=s^{*}$ with $D_{s} \equiv D$ and $D_{t} \equiv D^{*}$. (For example, $s$ and $s^{*}$ can be considered as the complex stereographic coordinates on $S^{2}$.)

The solution space of Eqs.(32) is four-dimensional, 8 the space of constants of integration, $\left(x^{a}\right)$; solutions can be written as

$$
Z=Z\left(x^{a}, s, s^{*}\right)
$$

We will be interested in several different spaces; the four dimensional space of the $\left(x^{a}\right)$; the space of

$$
\left(Z, Z_{s}, Z_{s^{*}}, Z_{s s^{*}}\right) \equiv\left(Z, D Z, D^{*} Z, D D^{*} Z\right) \equiv\left(Z, W, W^{*}, R\right)
$$

(defining the $\left.Z, W, W^{*}, R\right)$ and the six-dimensional space of $\left(Z, W, W^{*}, R, s, s^{*}\right)$.

Our starting equations are then rewritten

$$
\begin{aligned}
D^{2} Z & =S\left(Z, D Z, D^{*} Z, D D^{*} Z, s, s^{*}\right) \\
D^{* 2} Z & =S^{*}\left(Z, D Z, D^{*} Z, D D^{*} Z, s, s^{*}\right) .
\end{aligned}
$$

We identify the spaces

$$
\left(x^{a}\right) \Leftrightarrow\left(Z, W, W^{*}, R\right)
$$

for any fixed values of $\left(s, s^{*}\right)$, treating the relationship, Eq.(38), as a coordinate transformation between the two sets, that is parametrized by $\left(s, s^{*}\right)$. The six-space can then be coordinatized either by $\left(x^{a}, s, s^{*}\right)$ or by $\left(Z, W, W^{*}, R, s, s^{*}\right)$. It is useful to think of the larger space as being a two-dimensional bundle over the four-space, $x^{a}$. In our applications it is taken to be the sphere-bundle, physically, the bundle of null directions at each space-time point. This point of view will not be emphasized here.

We begin with the six gradient one-forms

$$
\theta^{i}=\left(\theta^{0}, \theta^{+}, \theta^{-}, \theta^{1}\right) \equiv \partial_{a}\left(Z, W, W^{*}, R\right) d x^{a}
$$




$$
\begin{aligned}
\theta^{0} & \equiv d Z-W d s-W^{*} d s^{*}=Z_{a} d x^{a}, \\
\theta^{+} & \equiv d W-D^{2} Z d s-D D^{*} Z d s^{*}=W_{a} d x^{a}, \\
\theta^{-} & \equiv d W^{*}-R d s-D^{* 2} Z d s^{*}=W_{a}^{*} d x^{a}, \\
\theta^{1} & \equiv d R-D^{*} D^{2} Z d s-D D^{* 2} d s^{*}=R_{a} d x^{a},
\end{aligned}
$$

$$
\begin{aligned}
\theta & \equiv d s, \\
\theta^{*} & \equiv d s^{*},
\end{aligned}
$$

and form the combinations $\omega^{i}=\left(\omega^{0}, \omega^{+}, \omega^{-}, \omega^{1}\right)$

$$
\begin{aligned}
\omega^{0} & =\theta^{0} \\
\omega^{+} & =\alpha\left(\theta^{+}+b \theta^{-}\right) \\
\omega^{-} & =\alpha\left(\theta^{-}+b^{*} \theta^{+}\right) \\
\omega^{1} & =\left(\theta^{1}+a \theta^{+}+a^{*} \theta^{-}+c \theta^{0}\right)
\end{aligned}
$$

and

$$
\begin{aligned}
\omega & =C \theta, \\
\omega^{*} & =C^{*} \theta^{*}
\end{aligned}
$$

where the $(\alpha, a, b, c)$ and $C$ are to be determined.

\section{A. Four-Dimensional Lorentzian Metrics}

From the four $\omega^{i}$, we form the 2-parameter, $\left(s, s^{*}\right)$ family, of Lorentzian four-metrics by

$$
\begin{aligned}
g\left(x^{a}, s, s^{*}\right) & =\omega^{0} \otimes \omega^{1}+\omega^{1} \otimes \omega^{0}-\omega^{+} \otimes \omega^{-}-\omega^{-} \otimes \omega^{+} \\
& =\eta_{i j} \omega^{i} \otimes \omega^{j}
\end{aligned}
$$

This defines a metric for each value of $s$ and $s^{*}$, such that the $\omega$ 's form a null tetrad. We wish to know how $(\alpha, a, b, c)$ should be specified for the 
$\left(s, s^{*}\right)$-dependent metrics to be "almost" conformally equivalent for all $\left(s, s^{*}\right)$. The metrics are said to be "almost" conformally equivalent if

$$
\begin{aligned}
D g & =U_{i j}\left[M\left[S, S^{*}\right]\right] \omega^{i} \otimes \omega^{j}+\Lambda\left[S, S^{*}\right] g, \\
D^{*} g & =U_{i j}^{*}\left[M^{*}\left[S, S^{*}\right]\right] \omega^{i} \otimes \omega^{j}+\Lambda^{*}\left[S, S^{*}\right] g,
\end{aligned}
$$

where, (1); $\Lambda$ and $\Lambda^{*}$ are explicit functions of $\left(S, S^{*}\right),(2) ; M\left[S, S^{*}\right]$ and $M^{*}\left[S, S^{*}\right]$ are specific non-linear functions of $\left(S, S^{*}\right)$ and their derivatives, [the "metricity expressions" or generalized Wunschmann conditions], (3); $U_{i j}[M]$ are functions of $M, D M$ and $D^{2} M$ that all vanish when $M=0 . S$ and $S^{*}$ are still arbitrary functions of $\left(Z, D Z, D^{*} Z, D D^{*} Z, s, s^{*}\right)$. The $\left(s, s^{*}\right)$ dependent metrics are conformally equivalent when $S$ and $S^{*}$ are such that $M\left[S, S^{*}\right]$ vanishes. For arbitrary $S$, however, the metrics are "almost" conformally related. We refer to (44) as the "minimal dependence conditions".

In this section we display the values of $(\alpha, a, b, c)$ in terms of $S$, that satisfy the Eqs.(44). We could do this by using, in principle a "simplicity" argument (i.e., by trying to do the simplest thing possible), which would consist of setting to zero certain components of $D g-\lambda g$, (for some $\lambda$ ); namely those that allow us to solve for $(\alpha, a, b, c)$ algebraically in terms of $\left(S, S^{*}\right)$ and their derivatives. The remaining components of $D g-\lambda g$ were then to be then shown to be of the form $U_{i j}\left[M\left[S, S^{*}\right]\right]$ depending on a single function $M\left[S, S^{*}\right]$ which vanish when $M\left[S, S^{*}\right]$ vanishes. In this manner, the unknown functions $(\alpha, a, b, c)$ were to be uniquely determined. In fact we did not do this. We did start this calculation and did, in this manner, determine, $(\alpha, a, b)$, (see below) but soon the complexity of the algebraic expressions and manipulations became unmanageable and we could not determine $c$ and $M\left[S, S^{*}\right]$ directly. There however was an alternative approach (See Sec.IV) that did allow us to finish the task.

In the following we will first state the main results (partially obtained by both methods) and then outline the "simplicity" argument. The results will then be discussed in subsection B. Finally, in Sec.IV, the alternative method will be described in detail. The equivalence of both methods is then shown.

The main results are the following determination of the unknown functions $(\alpha, a, b, c)$;

$$
b=\frac{1}{S_{R}^{*}}\left(\sqrt{1-S_{R}^{*} S_{R}}-1\right), \quad b^{*}=\frac{1}{S_{R}}\left(\sqrt{1-S_{R}^{*} S_{R}}-1\right),
$$




$$
\begin{aligned}
\alpha^{2}= & \frac{\left(\sqrt{1-S_{R}^{*} S_{R}}+1\right)}{2\left(1-S_{R}^{*} S_{R}\right)}=\frac{\left(1+b b^{*}\right)}{\left(1-b b^{*}\right)^{2}} \\
a= & \left(1-S_{R} S_{R}^{*}\right)^{-1}\left(1-\frac{1}{4} S_{R} S_{R}^{*}\right)^{-1}\left\{\frac{1}{2}\left[S_{W^{*}}^{*}+S_{W}^{*} S_{R}-T_{R}^{*}\right]\left(1+\frac{1}{2} S_{R}^{*} S_{R}\right)\right. \\
& \left.-\frac{3}{4} S_{R}^{*}\left[S_{W}+S_{W^{*}} S_{R}^{*}-T_{R}\right]\right\} \\
c= & -\frac{1}{2} G-\left(a-a^{*} b^{*}\right)\left(a^{*}-a b\right)\left(1+b b^{*}\right)^{-1}
\end{aligned}
$$

where $T \equiv D^{*} S, U \equiv D^{*} T=D^{2 *} S \equiv D^{2} S^{*}$, the subscripts on the $S, T, U$ refer to partial derivatives. $G$ is defined by

$$
\begin{aligned}
G\left(1+\frac{1}{2} S_{R} S_{R}^{*}\right)= & T_{W}+T_{W^{*}} S_{R}^{*}+T_{W^{*}}^{*}+T_{W}^{*} S_{R}-\frac{1}{2} U_{R} \\
& +\frac{1}{2}\left(S_{W}^{*} S_{W} S_{R}+S_{W^{*}} S_{W^{*}}^{*}+S_{W^{*}}^{*} S_{W^{*}} S_{R}^{*}+S_{W^{*}} S_{W}^{*}\right. \\
& \left.-S_{R}^{*} S_{Z}-S_{R} S_{Z}^{*}\right)-\frac{1}{2}\left(S_{W} S_{R}^{*}+S_{R} S_{W}^{*}+2 T_{R}^{*}\right) \frac{g^{1+}}{g^{01}} \\
& -\frac{1}{2}\left(S_{R} S_{W^{*}}^{*}+S_{W^{*}} S_{R}^{*}+2 T_{R}\right) \frac{g^{1-}}{g^{01}} .
\end{aligned}
$$

with

$$
\begin{aligned}
& \frac{g^{1+}}{g^{01}}\left(1-\frac{1}{4} S_{R} S_{R}^{*}\right)=-\frac{1}{2}\left[T_{R}-S_{W}-S_{W^{*}} S_{R}^{*}\right]+\frac{1}{4} S_{R}\left[T_{R}^{*}-S_{W^{*}}^{*}-S_{W}^{*} S_{R}\right] \\
& \frac{g^{1-}}{g^{01}}\left(1-\frac{1}{4} S_{R} S_{R}^{*}\right)=-\frac{1}{2}\left[T_{R}^{*}-S_{W^{*}}^{*}-S_{W}^{*} S_{R}\right]+\frac{1}{4} S_{R}^{*}\left[T_{R}-S_{W}-S_{W^{*}} S_{R}^{*}\right]
\end{aligned}
$$

The metricity expression is given by

$$
M\left[S, S^{*}\right]=\frac{-2}{\left(1-b b^{*}\right)}\left\{D b+S_{W^{*}}-b S_{W}-\left(S_{R}+b\right)\left(a^{*}-a b\right)\right\}
$$

with $b$ and $b^{*}$ given by Eq.(45).

Explicitly the simplicity argument is carried out as follows: We begin by constructing

$$
D g=\eta_{i j} D \omega^{i} \otimes \omega^{j}+\eta_{i j} \omega^{i} \otimes D \omega^{j} .
$$

Working out, (see appendix), via Eqs.(41) and (39), all the 


$$
D \omega^{i}=A_{j}^{i} \omega^{j},
$$

with $A_{j}^{i}$ explicit functions of the derivatives of $S$ and $S^{*}$ and the unknown functions $(\alpha, a, b, c)$, we obtain

$$
D g=\left[\eta_{k j} A_{i}^{k}+\eta_{i k} A_{j}^{k}\right] \omega^{i} \otimes \omega^{j} \equiv G_{i j} \omega^{i} \otimes \omega^{j},
$$

with symmetric $G_{i j}$. The $G_{i j}$ are thus also explicitly known functions (see appendix) of $S$ and $S^{*}$ and their derivatives and the $(\alpha, a, b, c)$. ( $D g$ should be thought of as the Lie derivative of the metric along the vector field defined by $(35)$.)

We now rewrite $D g$ by adding and subtracting a term $2 G_{01} \omega^{(+} \otimes \omega^{-)}$, obtaining

$$
\begin{aligned}
D g= & G_{01} g+G_{11} \omega^{1} \otimes \omega^{1}+2 G_{1+} \omega^{(1} \otimes \omega^{+)}+2 G_{1-} \omega^{(1} \otimes \omega^{-)} \\
& \left.+G_{--} \omega^{-} \otimes \omega^{-}+G_{++} \omega^{+} \otimes \omega^{+}+2\left(G_{01}+G_{+-}\right) \omega^{(+} \otimes \omega^{-}\right) \\
& +2 G_{0+} \omega^{(0} \otimes \omega^{+)}+2 G_{0-} \omega^{(0} \otimes \omega^{-)}+G_{00} \omega^{0} \otimes \omega^{0}
\end{aligned}
$$

A simple inspection of the explicit expressions for $G_{i j}=\left[\eta_{k j} A_{i}^{k}+\eta_{i k} A_{j}^{k}\right]$ and its conjugate (See the appendix) reveals that, contrary to the $2+1$ case of Section II, determining which combinations of them should vanish is not at all, in this case, obvious. However, guided by the procedure that leads to the Null Surface reformulation of GR, as explained in Section IV, we take the following steps.

1. We observe that $G_{11} \equiv 0$.

2. Setting

$$
G_{1+}[S, b, \alpha]=G_{1-}[S, b, \alpha]=0
$$

determines $b(S)$ and $\alpha(S)$ algebraically, as given in Eqs.(45) and (46).

3. If $b(S)$ is given as in Eqs.(45), then setting

$$
G_{++}[S, b(S), \alpha(S), a]=b^{* 2}(S) G_{--}[S, b(S), \alpha(S), a]
$$

allows us to determine the functions $a(S)$ algebraically as in Eq. (47).

4. We then have, (56), with (45) and (46), that

$$
\begin{aligned}
& G_{01}[S, b(S), \alpha(S), a(S)], \\
& G_{-+}[S, b(S), \alpha(S), a(S)], \\
& G_{--}[S, b(S), \alpha(S), a(S)]
\end{aligned}
$$


are now explicit functions of $S$ and moreover they satisfy

$$
\begin{aligned}
& G_{01}[S, b(S), \alpha(S), a(S)]+G_{-+}[S, b(S), \alpha(S), a(S)] \\
= & b^{*}(S) G_{--}[S, b(S), \alpha(S), a(S)]
\end{aligned}
$$

as an identity.

Up to this point, namely, imposing (55) and (56), we have that $D g$ is reduced to

$$
\begin{aligned}
D g= & G_{01} g+G_{--}\left(\omega^{-} \otimes \omega^{-}+b^{* 2}(S) \omega^{+} \otimes \omega^{+}+2 b^{*}(S)\left(\omega^{(+} \otimes \omega^{-)}\right)\right. \\
& +2 G_{0+} \omega^{(0} \otimes \omega^{+)}+2 G_{0-} \omega^{(0} \otimes \omega^{-)}+G_{00} \omega^{0} \otimes \omega^{0}
\end{aligned}
$$

where

$$
\begin{aligned}
G_{01} & =G_{01}[S, a(S)], \\
G_{--} & =G_{--}[S, b(S), \alpha(S), a(S)], \\
G_{0+} & =G_{0+}[S, b(S), \alpha(S), a(S), c], \\
G_{0-} & =G_{0-}[S, b(S), \alpha(S), a(S), c], \\
G_{00} & =G_{00}[S, b(S), \alpha(S), a(S), c, D c] .
\end{aligned}
$$

5. We now need to extract, from our minimal dependence condition (44), a linear combination of the remaining components of $D g-G_{01} g$ which, when vanishing, will allow us to obtain $c$ algebraically and simultaneously force $G_{0+}, G_{0-}$ and $G_{00}$ to vanish when $G_{--}[S, b(S), \alpha(S), a(S)]=0$. The linear combination of $G$ 's that determines $c$ in this way is

$$
\left(2-b S_{R}^{*}\right) G_{0+}+\left(S_{R}^{*}-2 b^{*}\right) G_{0-}+\alpha^{-1}\left(1-b b^{*}\right)\left(a S_{R}^{*}-S_{W}^{*}\right) G_{--}=0
$$

with $G_{0+}, G_{0-}$ and $G_{--}$given by (58).

This result becomes extremely difficult to see by simple inspection of the equations. Instead, one must turn to the methods of Sec.IV. From this analysis one could see that $c$ was given by (48) and that $G_{0+}, G_{0-}, G_{00}$ all vanish when $G_{--}=0$.

Based on this, we promote $G_{--}[S, b(S), \alpha(S), a(S)]$ to the metricity condition and make the following identifications: 


$$
G_{01}(S, a(S)) \equiv \Lambda(S)
$$

and

$$
G_{--}[S, b(S), \alpha(S), a(S)] \equiv M(S) .
$$

From this we then have the form of $G_{i j}$ given in our minimal dependence equation (44). When $M(S)=0$ we have that

$$
D g=\Lambda[S] g
$$

\section{B. Six Dimensional Metrics}

We now extend the metric $g$ to a six-dimensional metric by

$$
g^{(6)}\left(x^{a}, s, s^{*}\right)=g-\omega \otimes \omega^{*}=g-C C^{*} d s \otimes d s^{*}
$$

so that

$$
\begin{aligned}
D g^{(6)} & =D g-\left(C^{*} D C+C d C^{*}\right) d s \otimes d s^{*} \\
& =U_{i j}\left[M\left[S, S^{*}\right]\right] \omega^{i} \otimes \omega^{j}+\Lambda\left[S, S^{*}\right] g-\left(C^{*} D C+C D C^{*}\right) d s \otimes d s^{*},
\end{aligned}
$$

If the unknown $C$ is chosen so that $D C=\frac{1}{2} \Lambda C, D C^{*}=\frac{1}{2} \Lambda C^{*}$ then

$$
D g^{(6)}=U_{i j}\left[M\left[S, S^{*}\right]\right] \omega^{i} \otimes \omega^{j}+\Lambda g^{(6)} .
$$

This leads to the

Proposition 4 If the class of differential equations is restricted to those $S$ that satisfy the conditions

$$
M\left[S, S^{*}\right]=0, M^{*}\left[S, S^{*}\right]=0,
$$

then there exists on the six-space, a pair of conformal Killing fields $\xi=d / d s$ and $\xi^{*}=d / d s^{*}$. It is obvious that conformal factors $\Omega$ can easily be found for the six metric so that the conformal Killing fields become Killing fields and the six-space can be foliated with four-dimensional subspaces, ( $s$ and $s^{*}$ constant), with the induced four metrics all isometric. These four metric then map down to a unique conformal class of Lorentzian metric on the four-space of the $x^{a}$. 


\section{Relationship with the Null Surface Formulation of GR}

It is not hard to see that all Lorentzian 4-metrics (locally) are included in this construction. This follows from its equivalence to the Null Surface reformulation of General Relativity. In that work, 10 11 12 one begins with a four-manifold $\mathfrak{M}$ with an unknown - but to be determined - conformal Lorentzian metric and ask that there be a two-parameter, $\left(s, s^{*}\right)$, family of (local) null surface foliations of $\mathfrak{M}$ of sufficient generality, whose level surfaces are given by

$$
u=Z\left(x^{a}, s, s^{*}\right) .
$$

This requires that the unknown conformal metric satisfies

$$
g^{a b} \partial_{a} Z \partial_{b} Z=0
$$

for all $\left(s, s^{*}\right)$. The arbitrary conformal factor can depend on $\left(s, s^{*}\right)$. By repeated $\left(s, s^{*}\right)$ derivatives of Eq.(66), (explicitly, the following eight derivatives)

$$
D, D^{*}, D^{2}, D^{* 2}, D D^{*}, D^{*} D^{2}, D D^{* 2}, D^{2} D^{* 2},
$$

which with Eq.(66), yields nine relations so that the unknown conformal metric, $g^{a b}$, can be given completely in terms of a function

$$
S\left(Z, D Z, D^{*} Z, D D^{*} Z, s, s^{*}\right)
$$

that is defined by

$$
D^{2} Z=S, D^{* 2} Z=S^{*}
$$

and we are back to our starting point. These metrics satisfy our minimal dependence condition, Eq.(44), for $\left(s, s^{*}\right)$ dependence. If we continue and take the derivatives $D^{3}$ and $D^{* 3}$ of Eq.(66), we finally obtain the metricity conditions $M\left[S, S^{*}\right]=0, M^{*}\left[S, S^{*}\right]=0$.

Since we began with an arbitrary Lorentzian space we see that for any such space there exists an $S$ satisfying the metricity conditions that yields, via Eqs.(37), that metric up to conformal factor. 
It is now clear that the vacuum Einstein equations, with a specific choice of conformal factor, can be obtained by a further restriction on the class of functions $\left(S, S^{*}\right)$.

The procedure just outlined can be carried out explicitly, with considerable calculational effort, as follows:

Start with a function $Z=Z\left(x^{a}, s, s^{*}\right)$ and its $D, D^{*}$ and $D D^{*}$ derivatives as "primary" functions, i.e., $\theta^{i}=\left(\theta^{0}, \theta^{+}, \theta^{-}, \theta^{1}\right) \equiv\left(Z, W, W^{*}, R\right)$;

$$
\begin{aligned}
Z & =Z\left(x^{a}, s, s^{*}\right), \\
W & =D Z\left(x^{a}, s, s^{*}\right), \\
W^{*} & =D^{*} Z\left(x^{a}, s, s^{*}\right), \\
R & =D^{*} D Z\left(x^{a}, s, s^{*}\right)=D^{*} W=D W^{*} .
\end{aligned}
$$

It is assumed that these four relations can be inverted as $x^{a}=X^{a}\left(\theta^{i}, s, s^{*}\right)$. The set of "secondary" functions

$$
\begin{aligned}
S & \equiv D^{2} Z, \quad S^{*} \equiv D^{* 2} Z, \\
T & \equiv D R=D^{*} S=D^{*} D^{2} Z, \quad T^{*} \equiv D^{*} R=D S^{*}=D D^{* 2} Z, \\
U & \equiv D^{2} S^{*}=D^{* 2} S=D^{*} T=D T^{*}=D^{2} D^{* 2} Z,
\end{aligned}
$$

which can all be thought of as functions of $\left(Z, W, W^{*}, R, s, s^{*}\right)$ where the $x^{a}$ have been eliminated via the inversion of Eq. 676). The exterior derivatives (the space-time gradients, holding $\left(s, s^{*}\right)$ constant ) of the "primary" functions are

$$
d \theta^{i}=\partial_{a} \theta^{i} d x^{a}
$$

and for the "secondary" functions $S, T$ and $U$, they are given by

$$
\begin{aligned}
& d S=S_{Z} d Z+S_{W} d W+S_{W^{*}} d W^{*}+S_{R} d R=S_{\theta^{i}} d \theta^{i} \\
& d T=T_{Z} d Z+T_{W} d W+T_{W^{*}} d W^{*}+T_{R} d R=T_{\theta^{i}} d \theta^{i} \\
& d U=U_{Z} d Z+U_{W} d W+U_{W^{*}} d W^{*}+U_{R} d R=U_{\theta^{i}} d \theta^{i}
\end{aligned}
$$

If we write the unknown, but to be determined, inverse of the spacetime metric $g^{a b}\left(x^{a}, s, s^{*}\right)=\widehat{g}^{a b}\left(x^{a}\right) \omega^{2}\left(s, s^{*}\right)$, (i.e., where the $\left(s, s^{*}\right)$ behavior appears only in the conformal factor) as

$$
g^{I} \equiv g^{a b} \partial_{a} \otimes \partial_{b}
$$


then we can define the metric components in the gradient basis, $\theta_{a}^{i} \equiv \partial_{a} \theta^{i}$, by

$$
g^{i j}\left(x^{a}, s, s^{*}\right) \equiv g^{I}\left(d \theta^{i}, d \theta^{j}\right)=g^{a b} \theta_{a}^{i} \theta_{b}^{j} .
$$

Note the very important point that since $g^{a b}=\widehat{g}^{a b}\left(x^{a}\right) \omega^{2}\left(s, s^{*}\right)$ we have

$$
D g^{I} \equiv D g^{a b} \partial_{a} \otimes \partial_{b}=2 \omega^{-1} D \omega g^{I} \equiv \lambda g^{I}
$$

We, however, will not be using Eq.(72) fully until the end of the calculation. More explicitly, we will only be using different specific components of Eq.(72), i.e., specific components of

$$
D g^{\mathrm{I}}\left(d \theta^{i}, d \theta^{j}\right) \equiv D g^{a b} \theta_{a}^{i} \theta_{b}^{j}=\lambda g^{i j}
$$

along the way and only at the end, with the metricity condition, will the full Eq.(72) be used. Until this last condition is imposed the conditions on $D g^{I}$ are precisely our "minimal dependence conditions".

Starting with the condition that the level surfaces, $Z\left(x^{a}, s, s^{*}\right)=$ constant, (for each value of $\left(s, s^{*}\right)$ ) are null surfaces of the metric, we have that

$$
g^{00}=g^{\mathrm{I}}(d Z, d Z)=g^{a b} Z_{, a} Z_{,_{b}}=0 .
$$

By applying $D$ and $D^{*}$ to Eq.(74), we have

$$
\begin{aligned}
& D g^{a b} Z,_{a} Z,_{b}+2 g^{a b} W,_{a} Z,_{b}=0, \\
& D^{*} g^{a b} Z_{,_{a}} Z,_{b}+2 g^{a b} W_{, a}^{*} Z_{,_{b}}=0 .
\end{aligned}
$$

Thus from Eq.(74, using one component of Eq.(73)

$$
D g^{a b} Z,_{a} Z,_{b}=\lambda g^{a b} Z,_{a} Z,_{b}=0,
$$

we have

$$
\begin{aligned}
& g^{0+}=g^{I}(d D Z, d Z)=g^{I}(d W, d Z)=0, \\
& g^{0-}=g^{I}\left(d D^{*} Z, d Z\right)=g^{I}\left(d W^{*}, d Z\right)=0 .
\end{aligned}
$$

Next. applying $D$ to Eq.(79), yields 


$$
D g^{a b} W_{, a}^{*} Z_{,_{b}}+g^{a b}\left(R,_{a} Z_{,_{b}}+W_{,_{a}}^{*} W_{,_{b}}\right)=0 .
$$

Thus from Eq.(79), again with one component of Eq.(73)

$$
D g^{a b} W_{, a}^{*} Z_{,_{b}}=\lambda g^{a b} W_{, a}^{*} Z_{,_{b}}=0
$$

we have

$$
g^{\mathrm{I}}(d R, d Z)+g^{\mathrm{I}}\left(d W, d W^{*}\right)=0 \quad \Leftrightarrow \quad g^{-+}+g^{01}=0 .
$$

If $D$ and $D^{*}$ are applied respectively to Eqs.(78) and (79), we have

$$
\begin{aligned}
D g^{a b} W_{,_{a}} Z_{,_{b}}+g^{a b}\left(S,_{a} Z_{,_{b}}+W_{,_{a}} W_{,_{b}}\right) & =0, \\
D^{*} g^{a b} W_{,_{a}}^{*} Z_{,_{b}}+g^{a b}\left(S_{a}^{*} Z_{,_{b}}+W_{,_{a}}^{*} W_{,_{b}}^{*}\right) & =0 .
\end{aligned}
$$

Therefore, from Eq.(78) again using one component of Eq.(73),

$$
D g^{a b} W,_{a} Z_{,_{b}}=\lambda g^{a b} W,_{a} Z_{,_{b}}=0,
$$

(which implies its complex conjugate as well) then, using (70),

$$
\begin{array}{rlll}
g^{I}(d S, d Z)+g^{I}(d W, d W) & = & 0 & \Leftrightarrow g^{++}=-S_{R} g^{01} \\
g^{I}\left(d S^{*}, d Z\right)+g^{I}\left(d W^{*}, d W^{*}\right) & = & 0 & \Leftrightarrow g^{--}=-S_{R}^{*} g^{01}
\end{array}
$$

Continuing this process, i.e., applying $D$ and $D^{*}$ to Eq.(82), yields

$$
\begin{aligned}
& D g^{a b} W,_{a} W_{, b}^{*}+D g^{a b} Z,_{a} R,_{b}+g^{a b}\left(S,_{a} W_{, b}^{*}+2 W,_{a} R,_{b}+Z,_{a} T,_{b}\right)=0 \\
& D^{*} g^{a b} W,_{a} W_{, b}^{*}+D^{*} g^{a b} Z,_{a} R,_{b}+g^{a b}\left(W_{,_{a}} S_{, b}^{*}+2 W_{,_{a}}^{*} R,_{b}+Z,_{a} T_{, b}^{*}\right)=0 .
\end{aligned}
$$

Therefore, from Eq.(82) using two components of Eq.(73), we have

$$
D g^{a b} W_{,_{a}} W_{, b}^{*}+D g^{a b} Z,_{a} R,,_{b}=\lambda g^{a b}\left(W_{,_{a}} W_{,_{b}}^{*}+Z,_{a} R,,_{b}\right)=0,
$$

(and its complex conjugate) leads, respectively, to

$$
\begin{aligned}
g^{I}(d T, d Z)+g^{I}\left(d S, d W^{*}\right)+g^{I}(d R, d W)+g^{I}(d W, d R) & =0, \\
g^{I}\left(d T^{*}, d Z\right)+g^{I}\left(d R, d W^{*}\right)+g^{I}\left(d R, d W^{*}\right)+g^{I}\left(d W, d S^{*}\right) & =0 .
\end{aligned}
$$


Using Eqs.(70), (74), (78), (79) and (86), in (91) and (72) we obtain, respectively,

$$
\begin{aligned}
& 2 g^{+1}=-T_{R} g^{01}+S_{W} g^{01}+S_{W^{*}} S_{R}^{*} g^{01}-S_{R} g^{-1} \\
& 2 g^{-1}=-T_{R}^{*} g^{01}+S_{W^{*}}^{*} g^{01}+S_{W}^{*} S_{R} g^{01}-S_{R}^{*} g^{+1}
\end{aligned}
$$

which are easily solved for $g^{+1}$ and $g^{-1}$ :

$$
\begin{aligned}
g^{+1}\left(1-\frac{1}{4} S_{R} S_{R}^{*}\right)= & -\frac{1}{2}\left[T_{R}-S_{W}-S_{W^{*}} S_{R}^{*}\right] g^{01} \\
& +\frac{1}{4} S_{R}\left[T_{R}^{*}-S_{W^{*}}^{*}-S_{W}^{*} S_{R}\right] g^{01}, \\
g^{-1}\left(1-\frac{1}{4} S_{R} S_{R}^{*}\right)= & -\frac{1}{2}\left[T_{R}^{*}-S_{W^{*}}^{*}-S_{W}^{*} S_{R}\right] g^{01} \\
& +\frac{1}{4} S_{R}^{*}\left[T_{R}-S_{W}-S_{W^{*}} S_{R}^{*}\right] g^{01}
\end{aligned}
$$

Notice that Eqs. (91) and (92) are obtained just as well by taking $D^{*}$ of Eq. (86) and $D$ of (87) using Eq.(87) and two components of Eq.(73), we have

$$
D g^{a b} W_{, a}^{*} W_{, b}^{*}+D g^{a b} S,_{a} Z_{,_{b}}=0
$$

(with its complex conjugate).

Finally, by applying $D^{*}$ to Eq.(91), (or $D$ to Eq.(92)), we obtain the last metric component $g^{11}$;

$$
\begin{aligned}
-2\left(1+\frac{1}{2} S_{R} S_{R}^{*}\right) g^{11}= & \left(2 T_{W}^{*}+S_{W} S_{W}^{*}\right) g^{++}+\left(2 T_{W^{*}}+S_{W^{*}} S_{W^{*}}^{*}\right) g \\
& +g^{01}\left(U_{R}+S_{Z} S_{R}^{*}+S_{R} S_{Z}^{*}\right. \\
& \left.-S_{W^{*}} S_{W}^{*}-S_{W} S_{W^{*}}^{*}-2 T_{W^{*}}^{*}-2 T_{W}\right) \\
& +g^{-1}\left(S_{R} S_{W^{*}}^{*}+S_{W^{*}} S_{R}^{*}+2 T_{R}\right) \\
& +g^{+1}\left(S_{R} S_{W}^{*}+S_{W} S_{R}^{*}+2 T_{R}^{*}\right)
\end{aligned}
$$

using the equation

$$
D g^{a b}\left(T_{, a}^{*} Z_{,_{b}}+S_{, a}^{*} W_{,_{b}}+2 R,_{a} W_{,_{b}}^{*}\right)=0 .
$$


that arises from similar considerations, from Eq.(73), as before.

We emphasize that at this point we have not yet used the full set of components of Eq. (73) and consequently we do not yet have a single conformal metric by this construction - but instead we have an $\left(s, s^{*}\right)$ dependent family of conformal metrics. In other words we see that modulo an overall (conformal) factor, namely $g^{01}=\omega^{2}\left(s, s^{*}\right)$, a two parameter family of metrics, $g^{i j}\left(x^{a}, s, s^{*}\right)$, has been obtained by requiring that a series of $D$ and $D^{*}$ derivatives, applied to the null surface condition, Eq.(74) remains zero for all $\left(s, s^{*}\right)$ [this is the meaning of imposing Eqs. (77), (81), (85), (90), (96) and (98)]. All the components, in the gradient basis, $\theta_{a}^{i}$, have been expressed in terms of derivatives of $S$ and $S^{*}$. This $\left(s, s^{*}\right)$-dependent metric satisfies the minimal dependence condition, Eq.(44), and is, in fact, identical (up to a conformal rescaling) to the metric of Eq.(43), but is expressed in a gradient basis, rather than in the null tetrad basis. By using one further component of Eq.(73), namely applying $D$ to Eq.(86) and using

$$
D g^{a b}\left(W,_{a} W,_{b}+S,_{a} Z,_{b}\right)=0,
$$

we obtain the metricity condition the only condition of the functions $S$ and $S^{*}$. (Given below.) In this case the $\left(s, s^{*}\right)$ - dependent family of metric are all conformal to each other.

We notice that all the components of the metric, $g^{i j}$, are determined up to a single overall undetermined factor, namely $g^{01}$; i.e., $g^{i j}=g^{01} h^{i j}\left[S, S^{*}\right]$. To make the explicit comparison between this conformal metric and the metric, (43), we chose the special conformal gauge

$$
g^{01}=1
$$

If we take the null tetrad system, $\omega_{a}^{i}$ as a linear combination of the gradient basis $\theta^{j}{ }_{b}$ then re-expressing the metric in the null tetrad system, $\omega_{a}^{i}$, allows us to read off the coefficients, $(\alpha, a, b, c)$, of Eq.(41). Explicitly,

$$
g_{a b}=g_{i j} \theta^{i}{ }_{a} \theta^{j}{ }_{b}=\eta_{i j} \omega_{a}^{i} \omega^{j}{ }_{b}=\eta_{i j} K_{k}^{i} K_{l}^{j} \theta^{k}{ }_{a} \theta_{, b}^{l},
$$

or

$$
\eta_{k l} K_{i}^{k} K_{j}^{l}=g_{i j}
$$


where the coefficients $K_{j}^{i}$ are given by $\omega_{a}^{i} \equiv K_{j}^{i} \theta^{j}{ }_{a}$ and are shown explicitly in Eqs. (41). In particular, $\alpha=K_{+}^{+}, a=K_{+}^{1}, b=K_{-}^{+} / K_{+}^{+}$and $c=K_{0}^{1}$. From (101), then using the special conformal frame, $\left(g^{01}=1 \Rightarrow g^{+-}=-1\right)$, we obtain

$$
\begin{aligned}
b & =\frac{-1}{g^{--}}(\sqrt{J}-1) \\
b^{*} & =\frac{-1}{g^{++}}(\sqrt{J}-1) \\
\alpha^{2} & =\frac{g^{++} g^{--}}{2 J(\sqrt{J}-1)} \\
a & =\frac{g^{1+} g^{--}+g^{1-}}{J} \\
a^{*} & =\frac{g^{1-} g^{++}+g^{1+}}{J}, \\
c & =-\frac{1}{2} g^{11}+\frac{\left[g^{--} g^{1+}+g^{1-}+g^{1-} \sqrt{J}\right]\left[g^{++} g^{1-}+g^{1+}+g^{1+} \sqrt{J}\right]}{2 J(\sqrt{J}-1)}
\end{aligned}
$$

with

$$
J=\left(g^{-+}\right)^{2}-g^{++} g^{--}=1-S_{R} S_{R}^{*} .
$$

Since the components of the metric are functions of $S$ as obtained above, the parameters $(\alpha, a, b, c)$ are expressed in terms of $S$, as desired.

We can now see how this procedure justifies Eqs. (55), (56) and (59) used in Section III. Straightforward algebra shows that

$$
D g^{a b} \theta_{a}^{i} \theta_{b}^{j}=-\eta^{k l} G_{k m} \eta^{m n}\left(K^{-1}\right)_{l}^{i}\left(K^{-1}\right)_{n}^{j}
$$

Using (102) to translate Eqs. (77), (81), (85), (90), (96) and (98) in terms of $G_{i j}$, the following propositions follow:

Proposition 5 The vanishing of $D g^{a b} \theta_{a}^{0} \theta_{b}^{0}$ is equivalent to the vanishing of $G_{11}$. Explicitly,

$$
D g^{a b} \theta_{a}^{0} \theta_{b}^{0}=0 \Leftrightarrow G_{11}=0
$$

Proposition 6 The vanishing of $D g^{a b} \theta_{a}^{-} \theta_{b}^{0}$ and $D g^{a b} \theta_{a}^{+} \theta_{b}^{0}\left(\right.$ with $\left.G_{11}=0\right)$ is equivalent to the vanishing of $G_{1+}$ and $G_{1-}$. Thus

$$
D g^{a b} \theta_{a}^{-} \theta_{b}^{0}=0=D g^{a b} \theta_{a}^{+} \theta_{b}^{0} \Leftrightarrow G_{1+}=0=G_{1-} .
$$


Proposition 7 The vanishing of $D g^{a b}\left(\theta_{a}^{+} \theta_{b}^{-}+\theta_{a}^{0} \theta_{b}^{1}\right)$ and $D g^{a b}\left(\theta_{a}^{-} \theta_{b}^{-}+S_{R}^{*} \theta_{a}^{0} \theta_{b}^{1}\right)$ (with $G_{11}=G_{1+}=G_{1-}=0$ ) is equivalent to the vanishing of $\left[G_{++}-b^{* 2} G_{--}\right]$ and $\left[G_{+-}+G_{01}-b^{*} G_{--}\right]$.

$$
\begin{aligned}
D g^{a b}\left(\theta_{a}^{+} \theta_{b}^{-}+\theta_{a}^{0} \theta_{b}^{1}\right) & =D g^{a b}\left(\theta_{a}^{-} \theta_{b}^{-}+S_{R}^{*} \theta_{a}^{0} \theta_{b}^{1}\right)=0 \\
& \Leftrightarrow\left[G_{++}-b^{* 2} G_{--}\right]=\left[G_{+-}+G_{01}-b^{*} G_{--}\right]=(105)
\end{aligned}
$$

This justifies our choices of vanishing combinations of $G_{i j}$ in order to obtain $(\alpha, a, b, c)$ in terms of $S$ in Section III.

The metricity condition, from this point of view, is obtained simply by applying $D$ to Eq.(86), i.e. to

$$
g^{I}(d S, d Z)+g^{I}(d W, d W)=0,
$$

which, with the use of

$$
D g^{a b}\left(W,_{a} W_{,_{b}}+S,_{a} Z,_{b}\right)=0,
$$

which follows from the last component of (72), leads to

$$
g^{I}(d D S, d Z)+3 g^{I}(d S, d W)=0,
$$

or

$$
M\left(S, S^{*}\right)=\frac{1}{3}(D S)_{R}+S_{W} \frac{g^{++}}{g^{01}}+S_{W^{*}} \frac{g^{-+}}{g^{01}}+S_{R} \frac{g^{1+}}{g^{01}}=0 .
$$

Furthermore, with the use of (102) we can prove the following

Proposition 8 The vanishing of $D g^{a b}\left(\theta_{a}^{+} \theta_{b}^{+}+S_{R} \theta_{a}^{0} \theta_{b}^{1}\right)$ (with the earlier conditions) is equivalent to the vanishing of $G_{--}$. Thus if $G_{11}=G_{1+}=G_{1-}=0$, $G_{++}=b^{* 2} G_{--}$and $G_{+-}+G_{01}=b^{*} G_{--}$, then

$$
D g^{a b}\left(\theta_{a}^{+} \theta_{b}^{+}+S_{R} \theta_{a}^{0} \theta_{b}^{1}\right)=0 \Leftrightarrow G_{--}=0 .
$$

Note that $G_{--}=0$ implies $G_{+-}+G_{01}=0$.

This allows us to promote $G_{--}$to the status of metricity condition if $(\alpha, a, b, c)$ are given in terms of $S$, as argued in Section III.

When the metricity condition, Eq.(108), is imposed the expressions for the remaining components of Eq.(73) are identically satisfied and $G_{0-}$ and $G_{00}$ vanish. 
When $G_{--} \neq 0$, the expression from Eq.(44)

$$
U_{i j}\left[M\left[S, S^{*}\right]\right]
$$

is a linear combination of $M\left(S, S^{*}\right), D^{*} M\left(S, S^{*}\right)$ and $D^{2 *} M\left(S, S^{*}\right)$ which thus all vanish when $M\left(S, S^{*}\right)$ vanishes.

\section{Discussion}

In this work we have extended Cartan's beautiful construction of differential geometric structures that are naturally associated with ordinary differential equations, to a pair of overdetermined partial differential equations. The resulting geometric structures form a rich set of mathematical constructions that includes as a special case all Lorentzian space-times - and, as an obvious consequence, all solutions of Einstein's theory of general relativity.

The study of the Einstein equations via this approach had already begun in an earlier series of papers9.19.11.22 long before we connected it with Cartan's view. This work on general relativity is continuing with hopefully many applications, but the point of view towards it diverges from that of the present paper. Here we feel that the issues are more closely related to the study of equivalence classes of the starting equations under some set or class of transformations.

An immediate question that we wish to investigate is the following; given our set of equations

$$
D^{2} Z=S, \quad D^{* 2} Z=S^{*}
$$

where the $\left(S, S^{*}\right)$ satisfy the metricity conditions, the level surfaces of the (local) solutions, $u=Z\left(x^{a}, s, s^{*}\right)$, define a two-parameter family of null surfaces (a complete integral) in the space-time of the solution space, $x^{a}$, with the (conformal) metric, Eq.(43). In general, null surfaces develop caustics or wavefront singularities at which point the function $Z\left(x^{a}, s, s^{*}\right)$ no longer satisfies the differential equations - the local solutions break down. Nevertheless the space-time and its metric could be completely smooth there. Other families of null surfaces would exist that satisfied similar equations but with different $\left(S, S^{*}\right)$. We will consider the "restricted equivalence" problem to be the problem of finding all pairs of functions, $\left(S, S^{*}\right)$, that yield the same 
space-time metric. As a matter of fact, this "restricted problem" is intimately related with the equivalence problem under general contact transformations. A paper is being prepared on this issue.15

Another issue that we left untreated was how do the Cartan structure equations, Eq. (19), behave in the case of the pair of PDE's. It seems very likely that we will have the similar results to that obtained from the third order ODE of Sec.II, where the metricity function plays the role of a torsion tensor and we obtain a conformal connection rather than a metric connection. But this remains to be analyzed - the calculations being quite lengthy.

\section{Acknowledgments}

The authors thank the NSF for support under research grants \# PHY 92-05109, PHY 97-22049 and PHY 98-03301. Carlos Kozameh thanks CONICET for support.

ETN is endebted to Peter Vassiliou, Niky Kamran and Robert Bryant for enlightening discussions and a detailed proof of the four-dimensionality of the solutions space for our pair of pdes.

We thank Paul Tod for both pointing out to us the connection of our earlier work to the work of Cartan and Chern that got us started in this project, for his teaching us Cartan's construction for 2nd order ODE's and for his helpful comments on an early version of this manuscript. 


\section{References}

${ }^{1}$ E. Cartan, Les Espaces Generalises e L'integration de Certaines Classes d'Equations Differentielles, C.R. Acad.Sci., 206 , 1425-1429, (1938).

${ }^{2}$ E. Cartan, La Geometria de las Ecuaciones Diferenciales de Tercer Orden, Rev. Mat. Hispano-Amer. 4 ,1-31,(1941)

${ }^{3}$ E. Cartan, Sur une Classe d'Espaces de Weyl, Ann.Sc.Ec.Norm.Sup., 3e serie 60, 1-16, (1943)

${ }^{4}$ S-S. Chern, The Geometry of the Differential Equation $y^{\prime \prime \prime}=F\left(x, y, y^{\prime}, y^{\prime \prime}\right)$, in Selected Papers, Springer-Verlag, (1978), original (1940).

${ }^{5} \mathrm{~K}$. Wunschmann, Ueber Beruhrungsbedingungen bei Integralkurven von Differentialgleichungen, Inaug.Dissert., Leipzig, Teubner

${ }^{6}$ K.P. Tod, Einstein-Weyl Spaces and Third Order Differential Equations, J. Math, Phys., 41, 5572, (2000).

${ }^{7}$ R.L.Bryant, Proc.Symp.Pure.Maths. vol 53, pp33-88, (1991)

${ }^{8}$ Though there is a simple heuristic argument for the four-dimensionality of the solution space, recently, in a private communication, Peter Vassiliou and Niky Kamran have given a rigorous proof of this.

${ }^{9}$ S. Frittelli, C. Kozameh, E. Newman, GR Via Characteristic Surfaces, J. Math. Phys., 36, 4984, (1995).

${ }^{10}$ S. Frittelli, C. Kozameh, E. Newman, Lorentzian Metrics from Characteristic Surfaces, J. Math. Phys., 36 , 4975, (1995)

${ }^{11}$ S. Frittelli, C. Kozameh, E. Newman, Dynamics of Light cone Cuts of Null Infinity, Phys. Rev. D. 56 , 4729, (1997).

${ }^{12}$ S. Frittelli, C. Kozameh, E. Newman, Linearized Einstein Theory Via Null Surfaces, J. Math. Phys., 36 , 5005, (1995)

${ }^{13}$ D. Forni, M. Iriondo, C. Kozameh, Null Surface Formulation in 3D, J. Math, Phys., 41, 5517, (2000)

${ }^{14}$ M. Tanimoto On the Null Surface Formalism, gr-qc/9703003,,(1997) 
${ }^{15}$ S. Frittelli, N. Kamran, E. Newman, Differential Equations and Conformal Geometry, preprint, (2001)

\section{Appendix}

\section{A. Geometry of $\frac{d^{2}}{d s^{2}} \mathbf{Z}=\mathbf{E}\left(\mathbf{z}, \frac{d z}{d s}, \mathbf{s}\right)$}

We begin with an arbitrary function of four variables

$$
\Phi(z, s, u, v)=0
$$

assuming that it can be locally solved for any one of the variables. Considering the two two-spaces of $(z, s)$ and $(u, v)$, we see that a point, $(z, s)$ in the first, corresponds to a specific curve in the second - as well as the converse. If we solve Eq.(109) for

$$
z=z(s, u, v) \equiv z\left(s, x^{A}\right)
$$

by differentiating with respect to $s,(u, v)$ can be eliminated from the second derivative leaving

$$
z^{\prime \prime}=E\left(z, z^{\prime}, s\right) .
$$

The same thing can be done with the variables $(u, v)$ resulting in the second order differential equation

$$
\frac{d^{2} u}{d v^{2}}=U\left(u, \frac{d u}{d v}, v\right)
$$

for the curve in the $(u, v)$ space.

Cartan then asks for the conditions on $E\left(z, z^{\prime}, s\right)$ such that Eq.(111) is a geodesic for some (projective) symmetric connection - which is determined by the form of $E$. He find that $E\left(z, z^{\prime}, s\right)$ must satisfy

$$
\frac{d^{2}}{d s^{2}} E_{z^{\prime} z^{\prime}}-\frac{d}{d s} E_{z z^{\prime}}-E_{z^{\prime}} \frac{d}{d s} E_{z^{\prime} z^{\prime}}+2 E_{z z}+E_{z^{\prime}} E_{z z^{\prime}}-2 E_{z} E_{z^{\prime} z^{\prime}}=0
$$

and the connection is given, in the gradient basis, $z_{A} \equiv \partial_{A} z$ and $z_{A}^{\prime} \equiv \partial_{A} z^{\prime}$, by 


$$
\begin{aligned}
\nabla_{B} z_{A} & =0 \\
\nabla_{B} z_{A}^{\prime} & =2 \alpha_{(A}^{\prime} z_{B)}, \\
\alpha_{A}^{\prime} & =\frac{1}{2}\left(E_{z z^{\prime}}-\frac{d}{d s} E_{z^{\prime} z^{\prime}}\right) z_{A}+E_{z^{\prime} z^{\prime}} z_{A}^{\prime},
\end{aligned}
$$

remembering the projective equivalence $\Gamma_{B C}^{A} \sim \Gamma_{B C}^{A}+2 \delta_{(B}^{A} \Upsilon_{C)}$.

This arises from the following argument: If the curve determined in Eq.(110) by $(z, s)=$ constant has a tangent vector $t^{A}$, then $t^{A}$ has a vanishing product with the gradient of $z=z\left(s, x^{A}\right)$; i.e.,

$$
t^{A} z_{A}=0 \text {. }
$$

If $t^{A}$ is tangent to a geodesic then

$$
t^{B} \nabla_{B} t^{A}=\beta t^{A}
$$

and

$$
t^{B} \nabla_{B}\left(z_{A} t^{A}\right)=t^{A} t^{B} \nabla_{B} z_{A}+z_{A} t^{B} \nabla_{B}\left(t^{A}\right)=t^{A} t^{B} \nabla_{B} z_{A}=0 .
$$

Now since $z_{A}$ and $z_{A}^{\prime}$ form a basis set for the covectors, we have that

$$
\nabla_{B} z_{A}=a z_{(B} z_{A)}+b z_{(B}^{\prime} z_{A)}+c z_{(B}^{\prime} z_{A)}^{\prime}
$$

but from Eqs. (116) and (117) we have $c=0$ and hence

$$
\nabla_{B} z_{A}=a z_{(B} z_{A)}+b z_{(B}^{\prime} z_{A)}=\alpha_{(A} z_{B)}
$$

and immediately

$$
\nabla_{B} z_{A}^{\prime}=\alpha_{(A}^{\prime} z_{B)}+\alpha_{(A} z_{B)}^{\prime} .
$$

But via the projective equivalence they are the same as

$$
\begin{aligned}
& \nabla_{B} z_{A}=0, \\
& \nabla_{B} z_{A}^{\prime}=\alpha_{(A}^{\prime} z_{B)}
\end{aligned}
$$

By taking another $s$ (or prime) derivative

$$
\nabla_{B} z_{A}^{\prime \prime}=\alpha_{(A}^{\prime \prime} z_{B)}+\alpha_{(A}^{\prime} z_{B)}^{\prime}
$$


and comparing it with

$$
\begin{aligned}
\nabla_{B} z_{A}^{\prime \prime}= & \left(E_{z z} \nabla z_{A}+E_{z z^{\prime}} \nabla z_{A}^{\prime}\right) z_{B} \\
& +\left(E_{z z^{\prime}} \nabla_{A} z+E_{z^{\prime} z^{\prime}} \nabla_{A} z^{\prime}\right) z_{B}^{\prime}+E_{z^{\prime}} 2 \alpha_{(A}^{\prime} z_{B)}
\end{aligned}
$$

obtained from

$$
z^{\prime \prime}=E\left(z, z^{\prime}, s\right)
$$

and

$$
z_{A}^{\prime \prime}=E_{z} z_{A}+E_{z^{\prime}} z_{A}^{\prime},
$$

we recover Eqs.(112) and (115).

We are indebted to Paul Tod for explaining this construction, due to Cartan, to us.

B. $\mathbf{D} \omega=A \omega$

We have, via a lengthy calculation that the $A_{j}^{i}$, defined by $D \omega^{i}=A_{j}^{i} \omega^{j}$ or

$$
\begin{aligned}
D \omega^{0} & =A_{0}^{0} \omega^{0}+A_{+}^{0} \omega^{+}+A_{-}^{0} \omega^{-}+A_{1}^{0} \omega^{1} \\
D \omega^{+} & =A_{0}^{+} \omega^{0}+A_{+}^{+} \omega^{+}+A_{-}^{+} \omega^{-}+A_{1}^{+} \omega^{1}, \\
D \omega^{-} & =A_{0}^{-} \omega^{0}+A_{+}^{-} \omega^{+}+A_{-}^{-} \omega^{-}+A_{1}^{-} \omega^{1}, \\
D \omega^{1} & =A_{0}^{1} \omega^{0}+A_{+}^{1} \omega^{+}+A_{-}^{1} \omega^{-}+A_{1}^{1} \omega^{1},
\end{aligned}
$$

are given by

$$
\begin{aligned}
D \omega^{0}= & \left(1-b b^{*}\right)^{-1} \alpha^{-1}\left(\omega^{+}-b \omega^{-}\right) \\
D \omega^{+}= & \alpha\left\{S_{Z}-c\left(S_{R}+b\right)\right\} \omega^{0}+\omega^{1} \alpha\left\{S_{R}+b\right\} \\
& \omega^{+}\left(1-b b^{*}\right)^{-1}\left\{\left(1-b^{*} b\right) D \ln \alpha+S_{W}-b^{*}\left(D b+S_{W^{*}}\right)\right. \\
& \left.-\left(S_{R}+b\right)\left(a-a^{*} b^{*}\right)\right\} \\
& +\omega^{-}\left(1-b b^{*}\right)^{-1}\left\{D b+S_{W^{*}}-b S_{W}-\left(S_{R}+b\right)\left(a^{*}-a b\right)\right\} \\
D \omega^{-}= & \omega^{0} \alpha\left\{b^{*}\left[S_{Z}-c S_{R}\right]-c\right\}+\omega^{1} \alpha\left\{1+b^{*} S_{R}\right\} \\
& +\omega^{-}\left(1-b b^{*}\right)^{-1}\left\{\left(1-b b^{*}\right) D \ln \alpha-\left(a^{*}-a b\right)-b D b^{*}\right. \\
& \left.+b^{*}\left[S_{W^{*}}-b S_{W}-S_{R}\left(a^{*}-a b\right)\right]\right\} \\
& \omega^{+}\left(1-b b^{*}\right)^{-1}\left\{D b^{*}-\left(a-a^{*} b^{*}\right)\right. \\
& \left.+b^{*}\left[S_{W}-S_{W^{*}} b^{*}-S_{R}\left(a-a^{*} b^{*}\right)\right]\right\}
\end{aligned}
$$




$$
\begin{aligned}
D \omega^{1}= & \omega^{0}\left\{D c+T_{Z}+a S_{Z}-c\left(a S_{R}+a^{*}+T_{R}\right)\right\}+\omega^{1}\left\{a S_{R}+a^{*}+T_{R}\right\} \\
& \omega^{+}\left(1-b b^{*}\right)^{-1} \alpha^{-1}\left\{T_{W}+c+D a+a S_{W}\right. \\
& \left.-b^{*}\left(D a^{*}+a S_{W^{*}}+T_{W^{*}}\right)-\left(a S_{R}+a^{*}+T_{R}\right)\left(a-a^{*} b^{*}\right)\right\} \\
& \omega^{-}\left(1-b b^{*}\right)^{-1} \alpha^{-1}\left\{\left(D a^{*}+a S_{W^{*}}+T_{W^{*}}\right)\right. \\
& \left.-\left(a^{*}-a b\right)\left(a S_{R}+a^{*}+T_{R}\right)-b\left(T_{W}+c+D a+a S_{W}\right)\right\}
\end{aligned}
$$

C. $\mathbf{D} g=G_{i j} \omega^{i} \otimes \omega^{j}$

The calculation of the $G_{i j}$, defined by $\mathrm{D} g=G_{i j} \omega^{i} \otimes \omega^{j}$ begins with

$$
D \omega^{i}=A_{j}^{i} \omega^{j}
$$

and

$$
\begin{aligned}
g & =\eta_{i j} \omega^{i} \otimes \omega^{j} \\
D g & =\eta_{i j} D \omega^{i} \otimes \omega^{j}+\eta_{i j} \omega^{i} \otimes D \omega^{j}=\left\{\eta_{j k} A_{i}^{k}+\eta_{i k} A_{j}^{k}\right\} \omega^{i} \otimes \omega^{j} \equiv G_{i j} \omega^{i} \otimes \omega^{j}
\end{aligned}
$$

Then, by direct substitution, we have

$$
\begin{aligned}
D g\left(x^{a}, s, s^{*}\right)= & 2\left(A_{+}^{0}-A_{1}^{-}\right) \omega^{(+} \otimes \omega^{1)}+2\left(A_{-}^{0}-A_{1}^{+}\right) \omega^{(-} \otimes \omega^{1)} \\
& 2 A_{0}^{1} \omega^{0} \otimes \omega^{0}+2\left(A_{+}^{1}-A_{0}^{-}\right) \omega^{(0} \otimes \omega^{+)} \\
& +2\left(A_{-}^{1}-A_{0}^{+}\right) \omega^{(0} \otimes \omega^{-)}+2 A_{1}^{1} \omega^{(0} \otimes \omega^{1)} \\
& -2\left(A_{+}^{+}+A_{-}^{-}\right) \omega^{(+} \otimes \omega^{-)}-2 A_{-}^{+} \omega^{-} \otimes \omega^{-}-2 A_{+}^{-} \omega^{+} \otimes \omega^{+}
\end{aligned}
$$

with

$$
G_{i j}=\left\|\begin{array}{lllll}
i j & 0 & + & - & 1 \\
0 & 2 A_{0}^{1} & \left(A_{+}^{1}-A_{0}^{-}\right) & \left(A_{-}^{1}-A_{0}^{+}\right) & A_{1}^{1} \\
+ & \left(A_{+}^{1}-A_{0}^{-}\right) & -2 A_{+}^{-} & \left(A_{+}^{+}+A_{-}^{-}\right) & \left(A_{+}^{0}-A_{1}^{-}\right) \\
- & \left(A_{-}^{1}-A_{0}^{+}\right) & \left(A_{+}^{+}+A_{-}^{-}\right) & -2 A_{-}^{+} & \left(A_{-}^{0}-A_{1}^{+}\right) \\
1 & A_{1}^{1} & \left(A_{+}^{0}-A_{1}^{-}\right) & \left(A_{-}^{0}-A_{1}^{+}\right) & 0
\end{array}\right\|
$$

and explicitly,

$$
G_{11}=0
$$




$$
\begin{aligned}
G_{-1}= & -b\left(1-b b^{*}\right)^{-1} \alpha^{-1}-\alpha\left(S_{R}+b\right), \\
G_{+1}= & \left(1-b b^{*}\right)^{-1} \alpha^{-1}-\alpha\left\{1+b^{*} S_{R}\right\}, \\
G_{--}= & -2\left(1-b b^{*}\right)^{-1}\left\{D b+S_{W^{*}}-b S_{W}-\left(S_{R}+b\right)\left(a^{*}-a b\right)\right\}+\alpha\left(S_{R}+b\right), \\
G_{+-}= & \left(1-b b^{*}\right)^{-1}\left\{2\left(1-b^{*} b\right) D \ln \alpha+\left(1-b b^{*}\right) S_{W}-D\left(b b^{*}\right)\right. \\
& \left.\quad-\left(S_{R}+b\right)\left(a-a^{*} b^{*}\right)-\left(a^{*}-a b\right)\left[1+S_{R} b^{*}\right]\right\}, \\
G_{++}= & -2\left(1-b b^{*}\right)^{-1}\left\{D b^{*}-\left(a-a^{*} b^{*}\right)+b^{*}\left[S_{W}-S_{W^{*}} b^{*}-S_{R}\left(a-a^{*} b^{*}\right)\right]\right\}, \\
G_{01}= & \left(a S_{R}+a^{*}+T_{R}\right), \\
G_{0-}= & \left(1-b b^{*}\right)^{-1} \alpha^{-1}\left\{\left(D a^{*}+a S_{W^{*}}+T_{W^{*}}\right)-\left(a^{*}-a b\right)\left(a S_{R}+a^{*}+T_{R}\right)\right. \\
& \left.\quad-b\left(T_{W}+c+D a+a S_{W}\right)\right\}-\alpha\left\{S_{Z}-c\left(S_{R}+b\right)\right\}, \\
G_{0+}= & \left(1-b b^{*}\right)^{-1} \alpha^{-1}\left\{T_{W}+c+D a+a S_{W}-b^{*}\left(D a^{*}+a S_{W^{*}}+T_{W^{*}}\right)\right. \\
& \left.\quad-\left(a S_{R}+a^{*}+T_{R}\right)\left(a-a^{*} b^{*}\right)\right\}-\alpha\left\{b^{*}\left[S_{Z}-c S_{R}\right]-c\right\}, \\
G_{00}= & 2\left\{D c+T_{Z}+a S_{Z}-c\left(a S_{R}+a^{*}+T_{R}\right)\right\} .
\end{aligned}
$$

